\title{
6 Führung ist Beziehungsarbeit
}

\subsection{Wie gestalte ich gute Führungsbeziehungen?}

Alleine kann die beste Führungskraft nicht erfolgreich sein. Per Definition wäre sie nicht einmal eine Führungskraft, weil ihr die „Folger“ fehlen würden. Eine einzelne Person kann normalerweise nicht viel ausrichten. Sie braucht Leute, die am gleichen Strang ziehen und das richtige Umfeld. Alle Mitarbeiterinnen und Mitarbeiter werden für einen Erfolg gebraucht. Wenn viele gemeinsam in eine Richtung arbeiten, dann macht dies den Unterschied und bringt den Erfolg.

Gerade als Führungskraft müssen Sie bewusst kommunizieren. Tragfähige Arbeitsbeziehungen zu gestalten ist das A und O Ihrer Führungstätigkeit und dafür ist Kommunikation die Voraussetzung. Um professionell zu agieren, sollten Sie sich mit Kommunikation beschäftigen. $\mathrm{Zu}$ leicht geschieht es sonst: Jemand ist nicht gut drauf, ein falsches Wort fällt, schon ist der andere eingeschnappt. Ein Wort ergibt das andere. Die einst gute Beziehung ist schneller zerstört, als sie begonnen hat.

Grundvoraussetzung für eine funktionierende Führungsbeziehung ist, dass die Beteiligten im Erwachsenen-Ich-Zustand sind und sich gegenseitig im ErwachsenenIch-Zustand adressieren. Unter dieser Voraussetzung haben alle Beteiligten eine Haltung des „Ich bin OK - Du bist OK“. Auch wenn sie vielleicht nicht immer mit allem einverstanden sind, was die anderen tun, so wissen sie sich doch gegenseitig als Personen wertzuschätzen. Zu einer guten Beziehung gehört auch ein hoher Grad an individueller Autonomie. Damit verbunden ist die Wahlfreiheit zwischen verschiedenen Beziehungsrollen. Ich können und „dürfen“ sich beispielsweise eigenständig definieren, abgrenzen und alleine sein.

Ebenso wie Sie auch privat keine guten Beziehungen haben werden, wenn Sie selbst nicht „da“, nicht als Person im Hier und Jetzt präsent sind, funktioniert dies auch für professionelle Beziehungen nicht.

Nun kann es sein, dass Sie sich als Kind oder im Laufe der Jahre ein bestimmtes Repertoire an Beziehungsmustern zugelegt haben, die für Ihre Arbeit hinderlich sein können. Insofern ist es wertvoll, wenn Sie sich selbstkritisch hinterfragen, ob Sie bestimmte Beziehungsmuster haben.

Haben Sie wiederkehrende Beziehungsmuster, die Sie in bestimmten Situationen zeigen?

- Ziehen Sie sich bei Stress zurück?

- Können Sie „Intimität“ zulassen? Lassen Sie andere wirklich „nah“ an sich heran, zeigen Ihre wahren Gefühle und zeigen sich so, wie Sie sind?

- $\quad$ Reagieren Sie mit Rückzug, wenn Ihnen jemand zu „nah“ kommt? Stoßen Sie die Person dann zurück?

- $\quad$ Fühlen Sie sich leicht überlegen oder unterlegen?

- Wie strukturieren Sie Zeit?

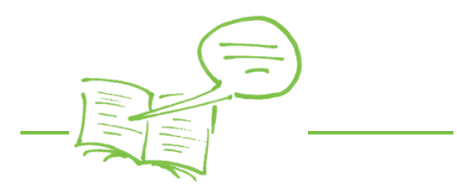

Zitat:

„Die Art und Weise, wie Menschen miteinander umgehen, wird zum entscheidenden Erfolgsfaktor.“

(Sprenger 2000, S. 186)

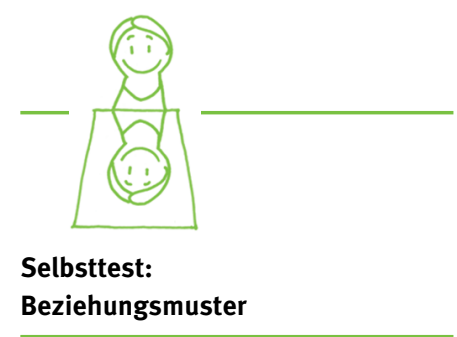




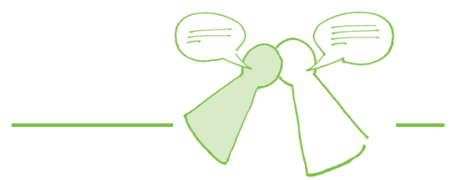

Beispiel:
Als Führungskraft ist es wichtig, dass Sie sich dessen bewusst sind, was und wie Sie kommunizieren. Wissen Sie selbst genau, was Sie kommunizieren möchten und Sind Sie selbst davon überzeugt? Sonst besteht eine hohe Wahrscheinlichkeit, dass das Ergebnis der Kommunikation unklar ausfallen wird und es schwierig wird, andere davon zu überzeugen.

Bei aller Bedeutung von guten Beziehungen und Mitmenschlichkeit am Arbeitsplatz sollten Sie nicht aus den Augen verlieren, dass es sich dabei immer um Arbeitsbzw. Führungsbeziehungen handelt. Sie sollten also darauf achten, dass Sie dabei nicht „aus der Rolle fallen“, sondern sich stimmig zu Ihrer Rolle als Führungskraft verhalten. Wenn Sie im Erwachsenen-Ich-Zustand bleiben und nicht in einen Kindoder Eltern-Ich-Zustand fallen, ist gewährleistet, dass Sie sich Ihrer Rolle bewusst sind und stimmig handeln (siehe auch Kap. 4.).

Für eine Führungsbeziehung ist es professionell, wenn Sie sich als Führungskraft abgrenzen können von anderen Leuten, von ihren Problemen, Erwartungen oder Konflikten. Können Sie dies nicht, so besteht die Gefahr, dass Sie aus Ihrer Rolle fallen und sich dann beispielsweise in eine symbiotische Arbeitsbeziehung hineinbegeben. Durch die Entwicklung Ihrer Autonomie bekommen Sie diese Abgrenzungsfähigkeit. Ihre Autonomie zu fördern ist demzufolge eine wichtige Entwicklungsaufgabe für Sie als Führungsperson.

\section{Fallbeispiel:}

Als absoluter Experte auf seinem Gebiet hatte Marco in der Bibliothek gewissermaßen eine informelle Führungsrolle inne. Er konnte auf einige feste Verbündete zählen, die dieselbe Wellenlänge hatten und zu ihm hielten. Dies hatte ihm bei seinen Projekten schon einige Male den Weg geebnet. Auf Katrin, die in der EDV-Abteilung eine Schlüsselposition besetzte, konnte er sich dabei stets verlassen. Nun war sie zu ihm gekommen, um ihn zu einem Problem um Rat zu fragen. Ganz im Gegensatz zu ihrer sonst guten Beziehung nahm das Gespräch eine plötzliche Wendung:

Marco: „Warum machst Du das denn nicht folgendermaßen ? Also, wenn Du das von Anfang an so gemacht hättest, dann wärst Du gar nicht erst in diese Schwierigkeiten gekommen. Warum hast Du es eigentlich nicht gleich so gemacht? Warum hast Du es denn erst so komisch gemacht?"

Katrin: „Danke für den tollen Rat! Das habe ich jetzt wirklich gebraucht! Dann frag’ ich dich halt nicht noch mal!“ Sagte es und stürmte hinaus.

Was war geschehen? Katrin hatte sich von Marco aus dem Eltern-Ich-Zustand angesprochen gefühlt und war unwillkürlich in eine trotzige Kind-Ich-Position gerutscht. Gerade als informelle Führungsperson hat Marco sich mit diesem Verhalten keinen Gefallen getan - und Katrin sich genau so wenig. Wäre Marco im Erwachsenen-Ich-Zustand gewesen, dann wäre er einfühlsamer mit ihr umgegangen und hätte vermutlich mehr Fragen gestellt. Falls er ihr eine Rückmeldung zu ihrem bisherigen Verhalten geben wollte, hätte er sie um Erlaubnis fragen sollen, anstatt ihr seine Meinung vor die Füße zu „knallen“. Er hätte ihr dann nicht ungefragt ihre Autonomie genommen. Vielleicht hätte sie am Ende sogar selbst eine Lösung zu ihrem Problem gefunden. 


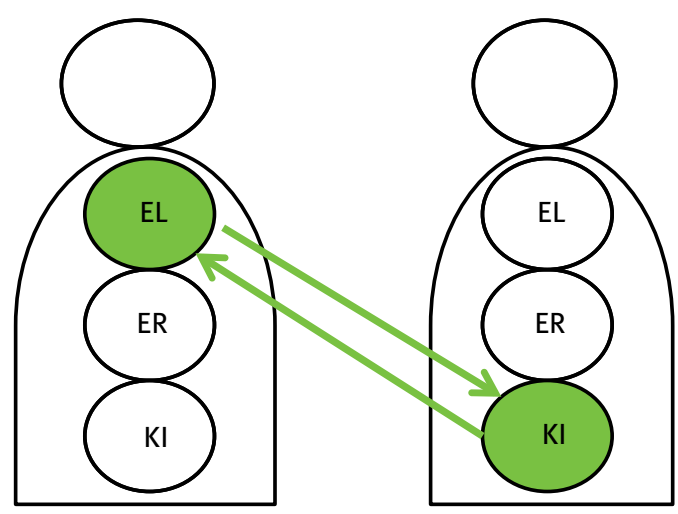

Abb. 12: Marco und Katrin im Fallbeispiel

Man darf bei der Betrachtung individueller Führungsbeziehungen und anderer Beziehungen am Arbeitsplatz nicht vergessen, dass die Beziehungen sich immer auch vor dem Hintergrund der Organisation entwickeln. Je nachdem, wie die Bibliothek gestrickt ist, beeinflusst sie die Beziehungen in ihrer Organisation in die eine oder andere Richtung. Wie soll sich beispielsweise ein Gefühl des Eingebunden seins und Dazugehörens in einer „kalten“ Organisation durchsetzen, in der die Menschen nicht zählen?

In sozialen Beziehungen geben sich die Menschen gegenseitig Zuwendung in den verschiedensten Formen, positive wie negative. Als Führungskraft ist es wichtig, dass Sie darauf achten, welche Art von Zuwendung Sie wem wofür geben. Andererseits ist es wichtig, dass Sie sich nicht von Zuwendung im beruflichen Kontext abhängig machen. Schließlich kann es immer wieder Zeiten geben, in denen nicht alle Ihre Entscheidungen voll mittragen, in denen es aber aus Ihrer Sicht keine andere Lösung gibt und Sie sich durchsetzen müssen. Würden Sie die Zuwendung, die Sie wie jede andere Person benötigen, überwiegend oder ausschließlich von Mitarbeiterinnen und Mitarbeitern, Kolleginnen und Kollegen oder Vorgesetzten beziehen, so hätten Sie vermutlich Schwierigkeiten damit, klare und sachdienliche Entscheidungen zu treffen, weil Sie eventuell befürchten würden, dass Ihre Entscheidungen bei den anderen nicht auf Gegenliebe stoßen.

Eine andere wichtige Aufgabe in diesem Zusammenhang ist es, dass sie bewusst entscheiden, wie Sie mit der Zuwendung anderer Menschen umgehen. Sie haben die Freiheit, Zuwendung anderer Personen abzulehnen, wenn sie Ihnen unpassend erscheint. Dieses gilt für positive Formen der Zuwendung ebenso wie für negative Formen. Sie müssen weder die Kritik anderer Personen zwangsläufig einstecken, noch Schmeicheleien oder deren Lob, wenn Ihnen das unpassend oder unaufrichtig erscheint.

Zur Führungsarbeit gehört es, selbst in einer passenden Form Zuwendung zu geben. Welche Formen von Zuwendung gibt es und welche Form ist passend?

Welche Art von Zuwendung Kinder von ihren Eltern oder anderen Bezugspersonen erhalten, ist von entscheidender Bedeutung dafür, was für eine Einstellung sie zu sich selbst und zu anderen Menschen entwickeln. Kommunikation ist im Allgemeinen mit dem Geben von Zuwendung verbunden. Diese kann sowohl verbal als auch nonverbal (beispielsweise ein Blickkontakt oder ein Lächeln) erfolgen. Menschliche Zuwendung lässt sich grob in vier verschiedene Qualitäten untergliedern:

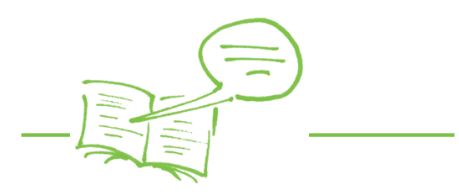

Zitat:

„Auch ein ehrlich wohlmeinender Paternalismus ist blind für den zugreifenden und bevormundenden Modus seiner Beziehung zum Menschen.“

(Sprenger 2000, S. 262)

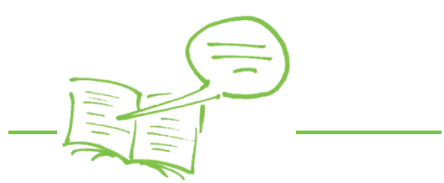

Zitat:

„Manch einer, der sich darüber beklagt, Arbeitskräfte würden heute nur noch ,jobben' und kein Geschäftsinteresse mehr zeigen, sollte zunächst scharf hingucken, wie es um die Qualität der menschlichen Beziehungen in seinem Verantwortungsbereich bestellt ist. Dieser Teil der Führungsaufgabe stellt allerdings spezifische Anforderungen: Freude am Kontakt mit Menschen; Gespür für die emotionale Lage anderer; aber auch eine gewisse Ruhe und etwas Zeit (....).“

(Doppler/ Lauterburg 2008, S. 47) 
- Unbedingte positive Zuwendung: Diese Zuwendung ist an keine Gegenleistung oder Erwartung von Gegenleistung gebunden. Es ist die Art von Zuwendung, die beispielsweise Babys in ihren ersten Lebensmonaten bekommen bzw. bekommen sollten: Das Gefühl angenommen und geliebt zu sein, so wie man ist, einfach weil man da ist. Das Vorhandensein unbedingter positiver Zuwendung festigt die Grundeinstellung des „Ich bin OK“. Das Fehlen dieser Art der Zuwendung führt hingegen meist zu einer „Ich bin nicht OK“-Einstellung. Bezogen auf eine Arbeitssituation könnte positive Zuwendung z.B. so aussehen: Eine Mitarbeiterin wird nach längerer Abwesenheit aufgrund einer Mutterschutzzeit von ihrer Vorgesetzten mit den Worten begrüßt: „Schön, dass Sie wieder da sind!“.

- Bedingte positive Zuwendung: Hier ist die positive Zuwendung mit einer bestimmten Bedingung, Verhaltensweise, Eigenschaft oder Fähigkeit verknüpft. So wird eine Grundeinstellung gefördert, die „Ich bin OK, wenn ich (eine bestimmte Arbeit gut erledige o.ä.). " Diese Form der Zuwendung bekommen wir von anderen Personen dafür, dass wir ihren Erwartungen entsprechen, aber nicht dafür, dass wir einfach so sind, wie wir sind. Somit wird mit der bedingten positiven Zuwendung die Anpassung an das soziale Umfeld gefördert.

- Bedingte negative Zuwendung: Diese Zuwendungsform funktioniert analog zur bedingten positiven Zuwendung. Ebenso wie diese dient sie der Anpassung an das soziale Umfeld. Wir bekommen sie, wenn wir nicht den Erwartungen anderer Menschen entsprechen, etwa wenn wir gegen geltende Normen verstoßen. Sie führt dazu, dass wir viel Beachtung erfahren, aber sie fühlt sich nicht wirklich gut an. Gefördert wird damit die Grundeinstellung „Ich bin nicht in Ordnung, wenn ich nicht (immer arbeite und nie krank werde; etwas Bestimmtes tue)." Kritische Hinweise und Strafen können als Maßnahmen für eine klare Grenzsetzung ebenfalls mit dieser Zuwendungsform verbunden sein. Für die Entwicklung eines realistischen Bildes von der eigenen Person haben bedingte negative Zuwendungen eine wichtige Funktion. Erhält ein Kind jedoch immer wieder nur bedingte negative Zuwendung, kann es sich nicht gut entwickeln, da die positiven Zuwendungsformen fehlen.

- Bedingungslose negative Zuwendung: Dies ist eine Zuwendungsform, die sehr problematisch ist, da sie sich allein destruktiv an eine Person richtet. Damit wird die Grundeinstellung „Ich bin nicht OK, egal was ich tue (am besten, es gäbe mich gar nicht)“ gefördert. Obwohl diese Form der Zuwendung so schädlich ist, kommt sie in der Kommunikation immer wieder vor. Manche Menschen suchen diese Form der Zuwendung geradezu, da sie ihnen immerhin eine gewisse Beachtung sichert, die sie auf anderem Wege sonst vielleicht nicht bekommen würden. Schließlich brauchen wir Zuwendung (vgl. Gührs/Nowak 2006, S. 85 ff.).

Je nachdem, welche Form der Zuwendung wir als Kind oft erhalten haben, kann es sein, dass wir auch später noch oft eine bestimmte Rolle einnehmen, um uns diese Form der Zuwendung zu sichern. Dazu gehören beispielsweise die Rolle des „Sorgenkindes“, des „Tollpatsches“, des „Klassenclowns“, des „Musterschülers“ oder des „Prügelknaben“ (vgl. Gührs/Nowak 2006, S. 90).

Bedingungslose negative Zuwendung sollte im Arbeitskontext, wie in jedem anderen Kontext, ein Tabu sein. Eine Person sollte immer mit Respekt für ihre Person behandelt werden, auch wenn er oder sie einen Fehler macht. Trotzdem Wertschätzung für die Person zu zeigen, aber ihr Verhalten zu kritisieren, also bedingte negative Zuwendung zu geben, ist eine geeignete Handlungsmöglichkeit für Fälle von Fehlverhalten. Wichtig ist es als Führungskraft, den Mitarbeiterinnen und Mitarbeitern bewusst bedingte und bedingungslose positive Zuwendung zu geben. Dadurch entsteht 
Vertrauen und auch dadurch, dass die anderen Personen darauf vertrauen können, dass sie keine komplette Ablehnung ihrer Person erleben werden, sondern, dass mit ihnen unter allen Umständen fair und wertschätzend umgegangen wird.

Dabei sollten Sie explizit darauf achten, dass Sie die verschiedenen Arten von Zuwendung verwenden, mit Ausnahme der bedingungslosen negativen Zuwendung. Manche Personen neigen dazu, nichts zu sagen, wenn alles gut läuft, aber sobald es irgendwo schlecht läuft, werfen sie mit negativer Zuwendung um sich. Wenn Fehler also die einzige Möglichkeit sind, die Aufmerksamkeit der Führungskraft zu erlangen, kann es zum einen demotivierend wirken, zum anderen bei manchen Personen geradezu ein schwieriges Verhalten „heranzüchten“. Sind Personen stark auf der Suche nach Zuwendung und sind durch ihre bisherige Entwicklung auf entsprechende Rollen getrimmt, so kann es sein, dass sie auch unter diesen Bedingungen den „Tollpatsch“ oder den „Prügelknaben“ „geben“, einfach um überhaupt Aufmerksamkeit zu erlangen. Als Führungskraft sollten Sie also darauf achten, dass Sie keine Fehlanreize setzen, indem Sie problematisches Verhalten „belohnen“.

Nur wenn Sie klar auftreten und klar kommunizieren, besteht eine realistische Chance, dass andere Ihnen folgen. Was brauchen Sie dafür?

1. Sich auf die Situation vorbereiten.

2. Überprüfen: Sind Sie präsent, im Hier und Jetzt?

3. Klarheit gewinnen I: Wer sind Sie? Wo sind Ihre Grenzen?

4. Klarheit gewinnen II: Was wollen Sie in dieser konkreten Situation?

5. Klar kommunizieren, was Sie wollen.

6. Andere mit ihren Wünschen einbeziehen.

Wenn Sie selbst innerlich klar sind, so wird es Ihnen leichter fallen, andere von Ihren Vorhaben zu überzeugen und gemeinsam erfolgreich in dieselbe Richtung zu ziehen. Es kommt also auf Ihren inneren Kompass an, auf Ihre Haltung. Ob Sie erfolgreich führen, hängt zwar nicht ausschließlich von Ihnen ab, aber ohne eine geeignete Haltung von Ihnen dazu, fehlt die Grundlage dafür, andere zu überzeugen. Der Grund für Misserfolg - der Konflikt bzw. dessen Wurzel - liegt dann in Ihnen selbst.

1. Sie sind im Erwachsenen-Ich-Zustand, haben eine „Ich bin OK - Du bist OK“-Haltung.

2. Sie sind autonom. Sie können sich professionell abgrenzen, ohne jedoch an Einfühlungsvermögen zu verlieren.

3. Guter Kontakt ist möglich, wenn Sie wirklich präsent im Hier und Jetzt sind und nicht nur physisch anwesend.

4. Sie achten darauf, ob die andere Person auch im Erwachsenen-Ich-Zustand ist.

5. Gegebenenfalls versuchen Sie, darauf hinzuwirken, dass sie aus einem Skriptverhalten herausfindet, indem Sie sie als OK und ebenbürtig behandeln und versuchen, mit Fragen ihr Erwachsenen-Ich und ihre Autonomie zu aktivieren. In jedem Fall nutzen Sie es nicht manipulierend aus, dass sie nicht im Erwachsenen-Ich-Zustand ist.

6. Treten Sie klar auf? Wissen Sie, was Sie kommunizieren wollen? Sind Sie selbst davon überzeugt?
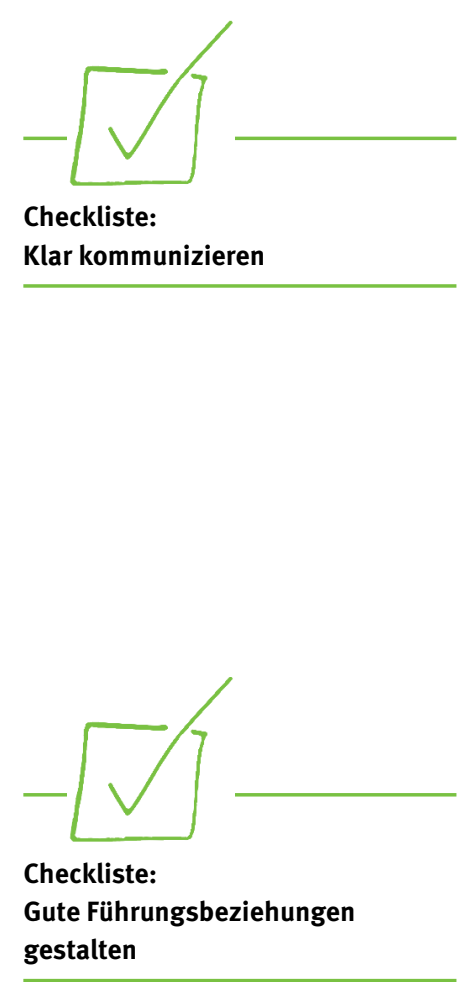


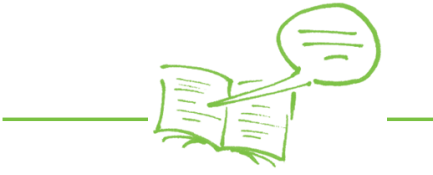

Zitat: „Was tue ich dazu, dass der andere sich verhält, wie er sich verhält?“ (Sprenger 2000, S. 90)

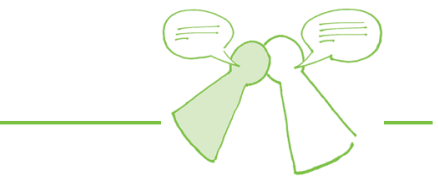

Beispiel:

\subsection{Wie verhalte ich mich stimmig bei schwierigen Führungsbeziehungen?}

Manchmal haben Sie vermutlich auch Führungsbeziehungen, die Sie als schwierig empfinden oder in denen Sie Ihr Gegenüber als schwierig empfinden. Vielleicht fühlen Sie sich dem nicht gewachsen oder die Situation ist festgefahren. Die Arbeit leidet darunter, Entscheidungen oder Innovationen stocken. Hilfreich ist es in all diesen Situationen, wenn Sie Ihre Rolle in der Führungsbeziehung selbstkritisch reflektieren.

Im Folgenden werden speziell herausfordernde Führungssituationen dargestellt.

\subsubsection{Ersatzgefühle - Jemand reagiert heftig oder unpassend}

Manchmal erleben wir, dass Menschen Gefühle zeigen, die nicht zur Situation passen, oder dass die Gefühle heftiger ausfallen, als es normal zu erwarten gewesen wäre. Oft halten solche Gefühle zudem über längere Zeit an.

Fallbeispiel:

Nora hatte sich in einer Sitzung von einer Kollegin schlecht behandelt gefühlt, weil diese eine Aussage zu ihrem Zuständigkeitsbereich getroffen hatte. Sie brodelte innerlich nach der Sitzung und sprach die Kollegin an. Diese entschuldigte sich sofort und schickte eine E-Mail zur Klarstellung an alle Beteiligten. Obwohl Nora mit dieser E-Mail im Prinzip einverstanden war, spürte sie weiterhin Wut in sich nagen und spielte in Gedanken verschiedene Szenarien durch, wie sie selbst es der Kollegin heimzahlte und auf ihrem Gebiet „wilderte“. Diese Gefühle tauchten über Tage immer wieder bei ihr auf. Nora begann sich selbst über ihre Wut und Rachsucht zu wundern. Rational konnte sie sich dies nicht erklären. Sie horchte mehr in sich hinein. Schließlich merkte sie, dass sie innerlich von der Angst erfüllt war, jemand könnte ihren Arbeitsbereich antasten.

Als Kinder lernen wir in unseren Familien zum Teil, dass bestimmte Gefühle gerne gesehen sind, andere abgelehnt werden. Da wir als Kinder vom Wohlwollen und der Zuwendung unserer Eltern abhängig sind, werden wir fortan bestrebt sein, Gefühle, die sie nicht sehen möchten, zu unterdrücken. Zu diesem Zweck überdecken wir diese ursprünglichen Gefühle dann mit anderen, unechten Gefühlen, den sogenannten „Ersatzgefühlen“. Als Kind war dies ein hilfreiches Verhalten. Legen wir es als Erwachsene später an den Tag ist es unproduktiv und zeigt, dass wir nicht im Erwachsenen-Ich-Zustand sind. Wir müssen dann zum Teil erst wieder erlernen, die ursprünglichen Gefühle zuzulassen (vgl. Stewart/Joines 2010, S. 27).

Ersatzgefühle lassen sich daran erkennen, dass sie länger andauern als „echte“, ursprüngliche Gefühle. Außerdem haben sie im Gegensatz zu ursprünglichen Gefühlen keine „produktive“ Funktion zur Lösung eines Problems. Es handelt sich dabei um Gefühle, die ein Unwohlsein bei uns hinterlassen und nicht wie funktionale Gefühle mit der Zeit abklingen. Vielmehr tauchen sie immer wieder auf und führen zu keiner Erleichterung. Sie bestehen auch dann noch fort, wenn der eigentliche Auslöser bereits abgestellt wurde (Gührs/Nowak 2006, S. 171 ff.).

Erleben wir ein Ersatzgefühl, drücken es aber nicht aus, sondern „sammeln“ es für später, so spricht man davon, dass wir eine „Rabattmarke“ gesammelt haben. 


\subsubsection{Negative Gefühle sammeln wie Rabattmarken}

\section{Fallbeispiel:}

Frau G., die Leiterin der Stadtbibliothek, wirkte fast immer freundlich im Umgang mit ihren Mitarbeiterinnen und Mitarbeitern. Selbst wenn sie ihr eröffneten, dass sie manche Arbeiten unmöglich übernehmen konnten, weil sie ihnen aus privaten Gründen nicht passten oder weil sie sich dafür nicht als genügend qualifiziert empfanden oder zu viel zu tun hätten, lächelte sie darüber hinweg und ertrug es mit stoischer Ruhe - auch wenn sie anderer Meinung war. Hin und wieder brach jedoch der still und heimlich aufgestaute Ärger darüber aus Ihr heraus und sie verschaffte sich Luft. Meistens traf es dann allerdings nicht die Personen, über die sie sich geärgert hatte, denn vor diesen hatte sie in gewisser Weise Angst. Vielmehr war es eine bestimmte Mitarbeiterin, Frau V., die normalerweise die aufgestaute Wut abbekam. Sie war eine sehr fleißige Mitarbeiterin, wohl so ziemlich die einzige, die ihr dauerhaft die Treue zu halten schien. Manchmal stellte sie sich allerdings etwas ungeschickt an. Das brachte Frau G.'s Fass dann zum Überlaufen. Frau V. nahm dies klaglos hin.

Als Analogie zum Sammeln von Treupunkten im Supermarkt wird in der Transaktionsanalyse vom psychologischen Sammeln von „Rabattmarken“ gesprochen. Hat eine Person in ihrem „Sammelheft“ eine gewisse Anzahl an Punkten gesammelt, so kann sie entscheiden, ob sie das Sammelheft einlösen möchte. Für das Einlösen des „Sammelheftes“ winkt ein „Preis“, dessen Größe davon abhängt, wie viele Rabattmarken gesammelt wurden. Eine Person, die oft eine kleinere Anzahl an Rabattmarken einlöst, bekommt nur einen kleinen Preis, während einer Person, die längere Zeit gesammelt hat, ein großer Preis für die Einlösung des Sammelheftes winkt. Da es bei den psychologischen Rabattmarken eine Wahl bezüglich des Zeitpunkts der Einlösung gibt, kann es sein, dass Sie Ihre Rabattmarken an einer ganz anderen Stelle einlösen und nicht dort, wo Sie sie gesammelt haben. Es trifft daher möglicherweise eine vollkommen unbeteiligte Person mit der Einlösung der Rabattmarken (vgl. Stewart / Joines 2010, S. 312). Immer wieder lässt sich beobachten, dass Menschen ungute Gefühle wie Wut sammeln, bis es irgendwann zum großen Ausbruch kommt.

Die Rabattmarken können vielfältiger Art sein, neben „Wutmarken“, die sich gegen andere, aber auch gegen die eigene Person richten können, beispielsweise „Depressionsmarken“. Manche Menschen sammeln ihre Rabattmarken über einen kurzen Zeitraum und brechen zur Einlösung einen Konflikt mit einem Kollegen vom Zaun. Andere hingegen sammeln über Monate, manchmal Jahre und lösen dann gleich einen ganzen Haufen von Rabattmarken auf einmal ein, um als Endauszahlung einen richtigen „Hauptgewinn“ zu bekommen. In schweren Fällen kann dies beispielsweise bin hin zur Entlassung, zum Magengeschwür, Herzinfarkt oder Suizid führen (vgl. Stewart/Joines 2010, S. 312 f.).

\subsubsection{Situationen werden wieder und wieder gleich „inszeniert“}

Vielleicht kennen Sie das: Eine Situation wiederholt sich in ähnlicher Weise immer wieder und Sie haben den Eindruck, „Dieses Spiel kenne ich doch.“? Immer wiederkehrende, zueinander passende Kommunikationsmuster von zwei oder mehr Personen, die sich periodisch wiederholen und immer wieder zu einem bestimmten Ergebnis führen, das schon von Anfang an vorausgesagt werden kann, werden als psychologische Spiele bezeichnet. Psychologische Spiele sehen nach außen hin zwar plausibel aus, haben aber verborgene Motive im Hintergrund. Sie sind stets mit einer Falle oder einem Trick verbunden. Als Nutzen erhalten die Spieler Zuwendung und eine Bestätigung ihrer eigenen Position.
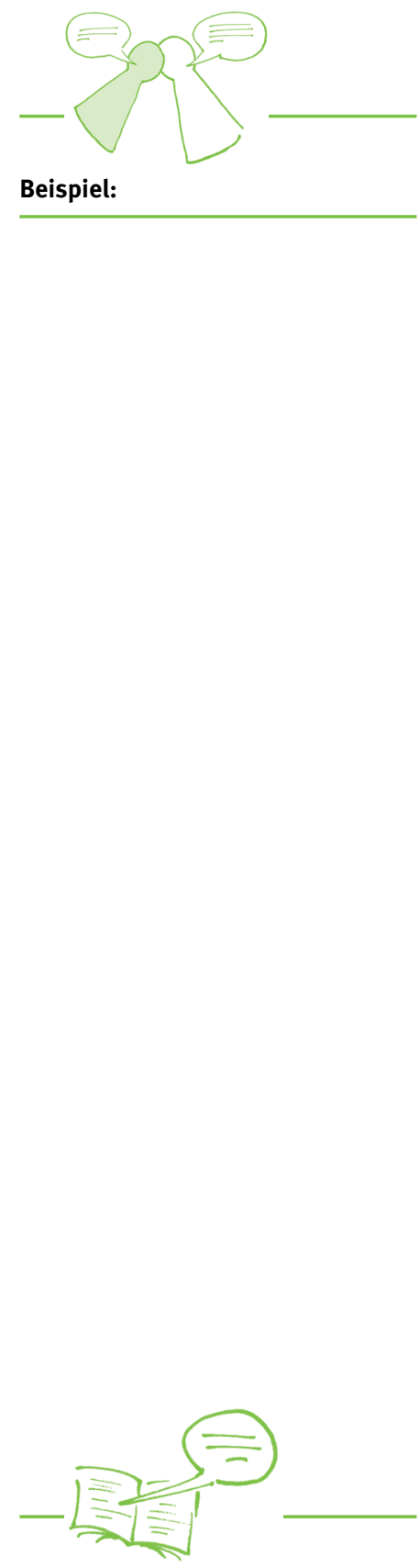

Zitat:

„Ein Spiel ist eine ständig wiederholte Abfolge von Transaktionen, bei der beide Seiten am Ende Maschengefühle (Anmerkung der Autorin: Ersatzgefühle) erleben. Dazu gehört immer ein Umschlag, ein Augenblick, wo beide Spieler erleben, daß etwas Unerwartetes und Unangenehmes passiert ist. Die Menschen spielen ihre Spiele, ohne dass sie sich das richtig klarmachen.“

(Stewart/Joines 2010, S. 27) 


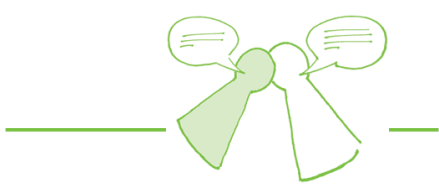

Beispiel:

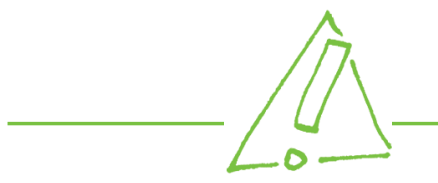

Tipp:

Bei Berne 2002 oder bei Gührs/ Nowak 2006, S. 193 ff.

werden verschiedene Formen psychologischer Spiele beschrieben.
Anders als das Wort „Spiel“ vermuten lassen könnte, führen psychologische Spiele nicht unbedingt zu Freude oder Vergnügen, sondern sind oft mit unangenehmen Gefühlen verbunden. (Berne 2002, S. $67 \mathrm{ff}$ )

\section{Fallbeispiel:}

„Wasch“ mir den Pelz, aber mach“ mich nicht nass,“ hatte Nora das Spiel von Herrn Dr. Schache getauft. Er spielte es folgendermaßen: Herr Dr. Schache forderte in mehr oder weniger regelmäßigen Abständen einen Wandel und mehr Innovationen in der Bibliothek. Es müsse einen „Ruck“ geben. In Sitzungen kündigte er an, dass es jetzt richtig losgehen würde. Voller Hoffnung war Nora dann aus den Sitzungen gegangen. Ihre Kolleginnen und Kollegen waren auch mehr oder weniger motiviert. Wenn es in der nächsten Sitzung dann darum ging, dass die Einzelnen konkrete Vorschläge dafür einbringen konnten, wie jetzt begonnen werden könnte, lautetet seine Antwort stets in etwa: „Ja, das ist eine gute Idee, aber es geht nicht, weil ..."

Mittlerweile hatte das Spiel dazu geführt, dass sich verschiedene verfeindete Gruppen in der Bibliothek gebildet hatten. Entscheidungen wurden nicht gefällt - oder nicht umgesetzt. Frustration machte sich allenthalben breit.

Im Fallbeispiel spielt Herr Dr. Schache ein „Ja, aber ...“-Spiel, u.a. im Arbeitskontext ein recht häufiges psychologisches Spiel. Mit verschiedenen Lösungsoptionen für ein Problem konfrontiert, lehnt der Initiator des Spiels alle Lösungsoptionen mit den Worten „Ja, aber “ ab. Dieses Verhaltensmuster kann er eine ganze Weile aufrechterhalten, bis seine Mitspieler letztlich entnervt aufgeben und er als „Sieger“ aus dem Spiel hervorgeht.

Das „Ja, aber ...“-Spiel ist nur eines von vielen psychologischen Spielen. Eine Reihe anderer Spielmuster sind oft im Arbeitskontext zu finden. Eine ausführliche Beschäftigung damit kann sehr erhellend sein.

Im Führungskontext ist es wichtig, nicht selbst in psychologische Spiele zu gelangen, da sie unproduktive Energie binden. Sie sollten hinterfragen: Inwiefern bin ich selbst anfällig für solche Spielmuster? Initiiere ich selbst psychologische Spiele? Lade ich andere dazu ein oder steige ich bei anderen Personen leicht in solche Spiele ein, wenn ich dazu eingeladen werde?

\subsubsection{Opfer-, Retter- und Verfolgerrollen}

Das Drama-Dreieck ist ein einfaches und wirkungsvolles Analyseinstrument für psychologische Spiele. Kennzeichen von psychologischen Spielen ist es, dass die am Spiel beteiligten Menschen bestimmte Rollen besetzen, die mit Skriptverhalten zusammenhängen: Die Rollen des Retters, des Opfers oder des Verfolgers. Diese drei Rollen können zeitgleich von drei verschiedenen Personen ausgefüllt werden oder tauchen zumindest im Laufe des Spiels alle auf:

- Opfer: Eine Person in der Opferrolle findet sich selbst unterlegen und nicht OK. Dies kann manchmal dazu führen, dass das Opfer sich einen Verfolger sucht, der ihm schadet, oder einen Retter, der ihm zur Hilfe kommt. Geschieht dies, wird das Opfer in seiner Auffassung bestätigt, dass es alleine nicht klar kommt.

- Retter: Für eine Person in der Retterrolle sind andere Personen nicht OK und ihr unterlegen. Aus seiner als überlegen wahrgenommenen Position bietet er ihnen daher Hilfe an.

- Verfolger: Eine Person in der Verfolgerrolle sieht andere Personen als nicht OK und als unterlegen an. Sie verfolgt andere, indem sie sie schlecht macht oder ihnen schadet. (Stewart / Joines 2010, S. 338 ff.) 
Menschen, die in den Rollen des Drama-Dreiecks handeln, befinden sich im Skriptverhalten und nicht im Hier und Jetzt des Erwachsenen-Ichs.

Bei psychologischen Spielen lässt sich in der Regel beobachten, dass eine Person ein Spiel in einer dieser drei Rollen beginnt und andere zum Mitmachen „einlädt“. Irgendwann im Laufe des Spiels findet ein plötzlicher Rollenwechsel bei dieser Person statt. Ein Beispiel hierfür wäre, dass jemand erst aus einer Opferposition agiert und, wenn sich ein hilfreicher Retter gefunden hat, irgendwann von einer Sekunde auf die nächste in eine Verfolgerrolle wechselt und den Retter schikaniert.

\section{Fallbeispiel:}

Frau G. sieht sich in einem heroischen Kampf. Sie will die Stadtbibliothek, die sie leitet, retten und auch die Mitarbeiterinnen und Mitarbeiter, die dort arbeiten. Sie rettet alles und jeden, egal, ob dies wirklich notwendig und erwünscht ist oder nicht. Jemand klagt über leiseste Anzeichen eines Schnupfens? Frau G. schickt sie nach Hause und übernimmt bereitwillig ihre Aufgaben, zusätzlich zu ihren eigenen. Denn sie hinterfragt weder die Notwendigkeit zum Retten, noch ob es ein passendes Verhalten für sie als Führungskraft ist, alles und jeden zu retten. Für ihre Mitarbeiterinnen und Mitarbeiter ist es oft sehr bequem, sich in eine Opferrolle zu begeben. Aber wehe, die Situation ändert sich und ihre eigenen Interessen sind in Gefahr! Dann kann es wie von Zauberhand passieren, dass sie ganz schnell ihre Opferrolle ablegen und Frau G. das Ziel ihres Unmutes wird. Als die Stadtbibliothek in neue Räumlichkeiten umzieht und in diesem Zuge das Rauchen in sämtlichen Räumen des Gebäudes verboten wird, stehen auf einmal drei aufgebrachte Kolleginnen vor Frau G. und machen ihr vor versammelter Mannschaft die Hölle heiß. Es könne doch wohl nicht angehen, dass sie sich nicht für ihre Interessen eingesetzt habe. Wie schwach wäre das denn? Wie könnte eine Leiterin zulassen, dass sie nun immer draußen rauchen müssten? Wie ein begossener Pudel steht Frau G. da und lässt die Vorwürfe auf sich einprasseln. Klein und hilflos fühlt sie sich in diesem Augenblick.

Wie unpassend ein solches Verhalten ist und wie schwierig es für eine Führungsbeziehung ist, wenn die Vorgesetzte sich gegenüber ihren Mitarbeiterinnen und Mitarbeitern klein und hilflos fühlt, dürfte auf der Hand liegen. Genauso unpassend ist natürlich das Verhalten der Mitarbeiterinnen und Mitarbeiter. Auch das, was dem Ganzen vorausgegangen war, nämlich dass Frau G. sich in ihrer Retterrolle gegenüber ihren Mitarbeiterinnen und Mitarbeitern überhöht hat, ist unpassend.

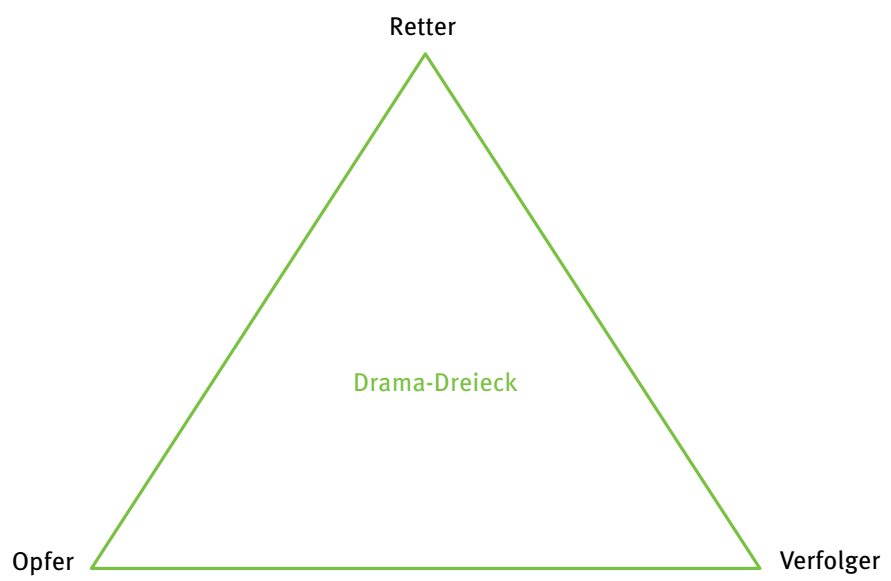

Abb. 13: Drama-Dreieck (modifiziert nach Stewart/Joines 2010, S. 340)

Wenn Sie sich selbst darüber bewusst werden, ob Sie standardmäßig zu bestimmten Rollen tendieren, kann Sie das in Zukunft davor bewahren, in die Komplikationen von psychologischen Spielen hineinzugeraten. Gerade in Führung ist dies wichtig,

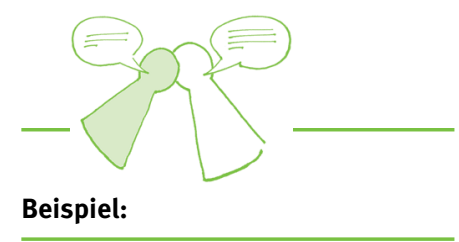




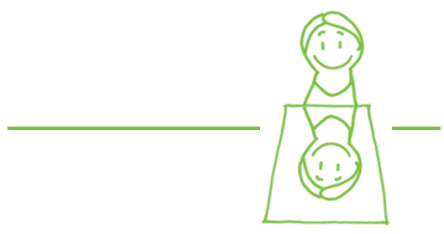

Selbsttest: Rollen im Drama-Dreieck denn als Teilnehmerin im Drama-Dreieck können Sie sonst allzu leicht in ein Verhalten abrutschen, das nicht stimmig für Ihre Rolle ist und dadurch Schaden anrichtet.

- Kommen Ihnen Situationen mit immer wiederkehrenden Kommunikationsmustern bekannt vor?

- $\quad$ Sind Sie in psychologische Spiele verwickelt?

- Welches ist Ihre bevorzugte Rolle im Drama-Dreieck?

- Kommt es regelmäßig vor, dass jemand Sie um Hilfe, Unterstützung oder Ratschläge bittet? Könnte es sein, dass diese Person sich in einer Opferposition befindet und den „Retter“ bzw. die „Retterin“ in Ihnen ansprechen möchte?

- Kommt es regelmäßig vor, dass jemand Sie schikaniert, herunterputzt, fertig macht oder Sie im Gegenteil gerne unterstützen möchte und Ihnen ungefragt Hilfe und Rat zukommen lässt? Möglicherweise sieht er oder sie Sie in einer Opferrolle oder möchte Sie dort gerne haben.

- Kommt es regelmäßig vor, dass Sie andere unsachlich kritisieren, auf ihnen herumhacken, sie schlecht machen? Möglicherweise sind Sie dann in einer Verfolgerrolle.

\subsubsection{Wie gehe ich gut mit privaten Problemen meiner Mitmenschen um?}

Besonderes Fingerspitzengefühl ist gefragt, wenn es um den Umgang mit privaten Problemen meiner Kolleginnen und Kollegen, Mitarbeiterinnen und Mitarbeiter geht. Die eigene Abgrenzungsfähigkeit und Beachtung der Ich-Zustände ist gerade in diesen Situationen von herausragender Bedeutung.

Einfach übergehen sollten wir Situationen nicht, in denen jemand private Probleme hat. Schließlich sollten wir den Menschen als Ganzes betrachten und können nicht nur einen Teil-Menschen als „Arbeitskraft einkaufen“! Sie müssen sich also darüber Gedanken machen, wie Sie mit solchen Situationen umgehen und gleichzeitig professionell und stimmig in Ihrer informellen oder offiziellen Führungsrolle sein können. Das gilt sowohl für Situationen, in denen jemand auf Sie zukommt und Sie um Hilfe oder Verständnis bittet, als auch für Situationen, in denen Sie als Führungskraft aktiv werden müssen, beispielsweise weil jemand alkoholabhängig ist oder in einer privaten Krise seine Arbeitspflichten grob vernachlässigt.

Um hierbei professionell zu agieren, müssen Sie sich professionell abgrenzen. Es ist also auch hier sehr wichtig, dass Sie sich selbst im Erwachsenen-Ich-Zustand befinden, um Ihre Rolle zu wahren. Dann widerstehen Sie auch leicht der Versuchung, in eine Retterrolle und damit ins Drama-Dreieck eingeladen zu werden.

Aber wie kann ich überhaupt helfen, ohne selbst zum Retter zu werden? Nun, ein Teil der Lösung ist sicherlich schon einmal dadurch erreicht, dass Sie selbst im Erwachsenen-Ich-Zustand sind. Aus diesem Zustand heraus können Sie dann etwas machen, was in der Transaktionsanalyse als Vertragsarbeit bezeichnet wird: Mit Ihrem Gegenüber klären, was er oder sie von Ihnen möchte, wie Sie ihm oder ihr helfen könnten - und bei sich selbst zu schauen, ob Sie das machen können und möchten, was er oder sie von Ihnen möchte. Außerdem kann es schon sehr helfen, wenn Ihr Gegenüber Ihre Zuwendung spürt, wenn er oder sie merkt, da interessiert sich jemand für mich, sogar wenn nicht alles gut ist. 


\subsection{Mit Klarheit Konflikte spielerisch lösen}

Leider ist die Welt nicht immer ideal. Allzu oft gibt es Konflikte bei der Arbeit. Was ist, wenn die Beziehung bereits Schaden genommen hat und es einen Konflikt gibt?

\section{Fallbeispiel:}

Nora zuckte noch immer zusammen, wenn sie an das Scheppern der Tür dachte. Niemals zuvor hatte ein solcher Lärm die Ruhe in dieser Bibliothek durchschnitten.

„Macht doch, was ihr wollt“, hatte Herr 0. gebrüllt, bevor die Tür zuflog. „Ich werde hier ja eh nicht mehr gebraucht.“

Vorausgegangen war ein Gespräch, bei dem auch die Leiterin der Benutzung anwesend war. Im Mittelpunkt des Gesprächs hatte die Abschaffung eines speziellen Ausleih- und Beratungsservices für ein befreundetes Forschungsinstitut vor Ort in einem anderen Stadtteil gestanden. Ein Steckenpferd von Herrn 0., das er ursprünglich ins Leben gerufen hatte - und wohl so ziemlich das Einzige, was er hier voller Leidenschaft tat. 24 Jahre war das nun her, und angesichts geänderter Rahmenbedingungen war dieser Service nicht mehr zeitgemäß. Nora wollte ihn daher im Zuge eines Auskunftsservices über Facebook, an dem sie arbeitete, einstellen.

Hier ist das Vorliegen eines Konflikts offensichtlich, aber es gibt auch subtilere Anzeichen, beispielsweise bei verdeckten Konflikten. Woran können Sie sonst erkennen, dass ein Konflikt vorliegt?

Mögliche Zeichen für Konflikte sind beispielsweise:

- „Mauern“.

- Dienst nach Vorschrift.

- Prioritäten werden verändert.

- Es ist schwierig, einen gemeinsamen Termin zu finden.

Konflikte sind ein normaler Bestandteil unseres Lebens. Gerade im Kontext von Wandel, der Einführung neuer Angebote und Prozesse bzw. der Veränderung oder Abschaffung vorhandener sind Konflikte vollkommen natürlich. Ein Alarmzeichen wäre eher das völlige Ausbleiben von Konflikten. Stehen Änderungen an, aber es treten keine Konflikte offen zutage, so erscheint dies zunächst bequem. Aber spiegelt es die Realität wider?

Folgende Punkte könnten Sie abprüfen, wenn Konflikte, mit denen Sie gerechnet haben, nicht zutage treten:

- Sind wirklich alle einer Meinung?

- $\quad$ Aus welchen Ecken wären eigentlich Widerstände zu erwarten?

- Bei welchen Themen wären eigentlich Widerstände zu erwarten?

- Woran könnte es liegen, dass Widerstände nicht in Erscheinung treten?

- Was kann getan werden, um den Konflikt oder die Konflikte offen auszutragen und dafür zu sorgen, dass nichts im Verborgenen schwelt und dort womöglich großen Schaden anrichtet?

Obwohl - oder gerade weil - die meisten von uns eher bestrebt sind, Konflikte zu vermeiden, kann es sehr hilfreich sein, wenn wir sie als Chance begreifen. Durch eine konstruktive Lösung können Dinge geklärt werden und Beziehungen auf eine neue Basis gestellt werden. Kaum etwas ist Kraft raubender als ein dauerhaft schwelender Konflikt, dem immer aus dem Weg gegangen wird, und kaum etwas kann derart viel Kraft freisetzen, wie die Bewältigung oder Klärung eines Konfliktes. Die Klärung von Beziehungen und Standpunkten kann ein gutes Gefühl des „aufgeräumt seins“ bei

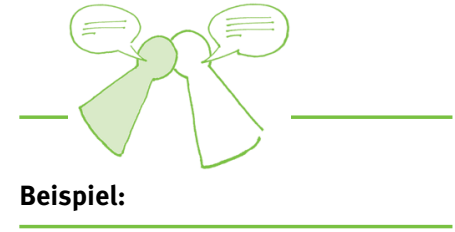

Beispiel:

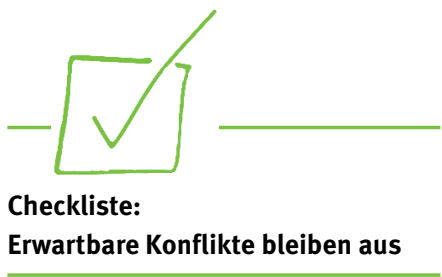




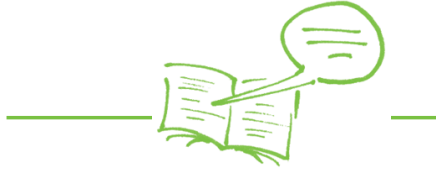

Zitat:

„Konstruktive Konfliktaustragung

kann zur Lösung von sachlichen

Problemen führen, neue

Perspektiven eröffnen, kreatives

Verhalten stimulieren, kann

Veränderungen von Personen, von

Wertvorstellungen und letztendlich

der Gesellschaft bewirken."

(Hintz 2011, S. 117) uns hinterlassen und uns die Power geben, neu durchzustarten - sogar wenn wir am Ende immer noch verschiedener Meinung sind. Die Klärung von Standpunkten und die anschließend vorhandene gemeinsame Basis für Austausch und Kommunikation, für die Fortsetzung des Dialogs sowie der Beziehung, geben uns die Chance, auf einer ehrlichen und authentischen Basis die Beziehung in der Zukunft weiterzuknüpfen. Wir können gemeinsam weiterarbeiten und vielleicht eines Tages wieder in größerer Übereinstimmung an gemeinsamen Zielen zu arbeiten. Bei einem dauerhaft fortgesetzten Konflikt oder einem mit großer Aufregung inszenierten Beziehungsabbruch würde die Basis hierfür fehlen. Kraft geht verloren und die Produktivität verzeichnet starke Einbußen. Alle Seiten stehen am Ende als Verlierer da.

In der Praxis werden Konflikte allerdings häufig verdrängt oder vermieden, anstatt sie konstruktiv anzugehen. Würde etwa der im Fallbeispiel bestehende Konflikt mit Herrn O. im weiteren Verlauf verdrängt, so könnte dies die Einführung des Facebook-Auskunftsservices torpedieren. Herr O. würde sich weiterhin um sein nicht mehr zeitgemäßes Steckenpferd kümmern und seine Arbeitskraft würde im Prinzip „verschwendet" werden, weil sich die ohnehin geringe Nachfrage nach diesem Service schon seit Jahren auf einem absteigenden Ast befindet. Gleichzeitig würde Herr O. sich vermutlich gekränkt und nicht wertgeschätzt fühlen und möglicherweise auf Rache sinnen.

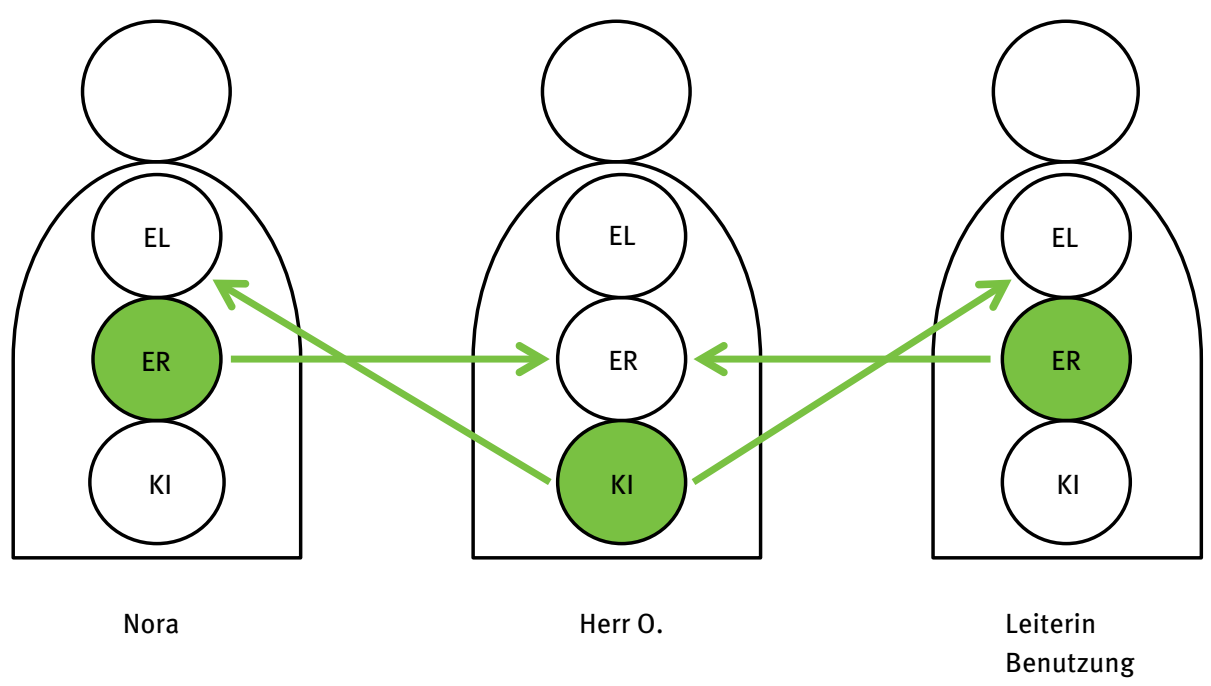

Abb. 14: Ich-Zustände während des Konflikts im Fallbeispiel

Ein konstruktiver Umgang mit Konflikten ist somit von großem Wert. Welche Möglichkeiten gibt es, um in der Praxis konstruktiv mit auftauchenden Konflikten umzugehen? 


\subsubsection{In sechs Schritten Konflikte lösen}

Im Folgenden wird ein sechsteiliger Prozess zur systematischen Konfliktlösung vorgestellt.

\section{Schritt: Zur Ruhe kommen, entspannen, äußerlich Abstand gewinnen}

Dies ist der erste und wichtigste Schritt. Sie sollten ihn auf keinen Fall auslassen! Wird dieser Schritt übersprungen, wird eine gute Konfliktlösung schwierig: Suchen Sie erst wieder das Gespräch, wenn Sie sich beruhigt haben. So vermeiden Sie auch, sich um Kopf und Kragen zu reden!

Fallbeispiel:

Herr O. spürte die Wut in sich hochkochen, ist aber in der Konfliktsituation geblieben.

Hilfreich wäre es im Fallbeispiel gewesen, wenn Herr O. sich rechtzeitig zurückgezogen hätte, um im räumlichen Abstand zur Ruhe zu kommen. Er hätte sich etwa mit den Worten verabschieden können: „Ich denke, wir finden jetzt keine gemeinsame Linie. Lassen Sie uns das Gespräch bitte vertagen." Auch Nora oder die Benutzungsleiterin hätten dies anbieten können, bevor die Situation eskalierte. Jetzt im Nachhinein erscheint es am besten, wenn sie sich erst wieder ins Gespräch begeben, wenn sich alle Gemüter abgekühlt haben. Bis dahin sollten sie sich auch räumlich, soweit es geht, voneinander entfernt halten. Es hilft, mindestens eine Nacht darüber schlafen, sich etwas Gutes zu tun und zu entspannen.

Insbesondere wenn die Situation emotional aufgeladen ist: Beenden Sie das Gespräch, entfernen Sie sich aus der Situation, gewinnen Sie Abstand - am besten auch räumlich.

Notizen für Ihre Konfliktlösung:
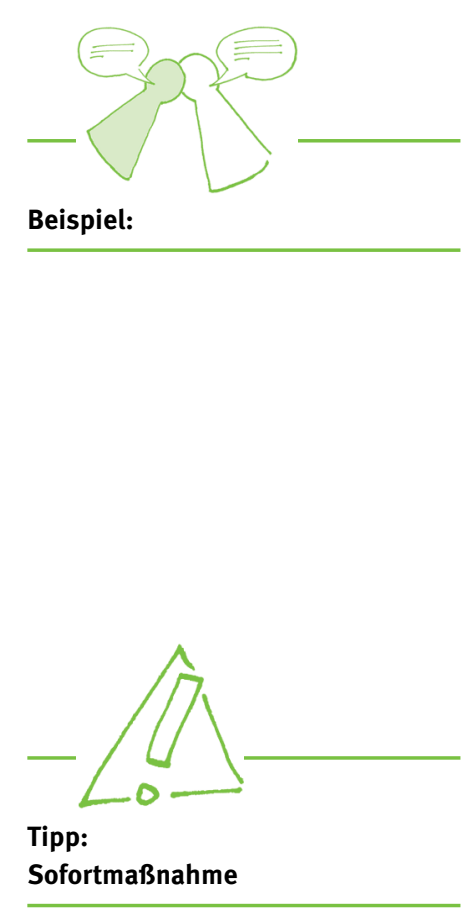

\section{Schritt: Innerlich Abstand gewinnen}

Dem äußerlichen Abstand sollte das Gewinnen eines innerlichen Abstands folgen. Sie sollten zur Ruhe kommen und sich auf sich selbst besinnen. Denn bei Ihnen selbst liegt auch der Startpunkt, wenn Sie wieder in die Konfliktlösung einsteigen möchten. 


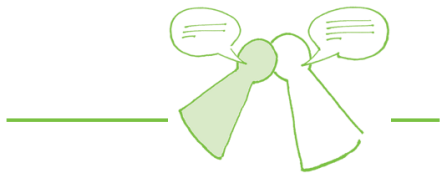

Beispiel:

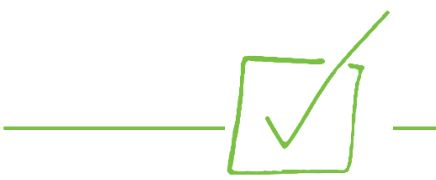

Checkliste:

Innerlich Abstand gewinnen im Konfliktfal

Fallbeispiel:

Alle Beteiligten haben sich räumlich zurückgezogen und gehen gewissermaßen in Klausur mit sich selbst. Nora überlegt, was der Konflikt mit ihr zu tun hat, warum sie sich so betroffen fühlt und inwiefern dabei ihre eigenen Werte und Grenzen verletzt wurden.

Sie besinnen sich auf sich selbst:

_ _Wer bin ich?" So definieren Sie sich.

_ _Wer bist Du?“ Setzen Sie Ihre „Brille“ mit vorhandenen Erfahrungen und Denkmustern ab. Betrachten Sie Ihr Gegenüber unvoreingenommen.

- Wo wurden zwischen Ihnen Grenzen verletzt?

_ Welche Grenzen möchten Sie bewusst wieder setzen?

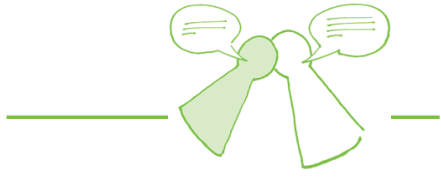

Beispiel:

\section{Schritt: Die Situation mit Ruhe und Abstand analysieren}

Als nächster Schritt folgt nun eine Analyse der Situation. Dafür ist es wichtig, ruhig und im Hier und Jetzt zu sein.

Gelingt es nicht, Ruhe und Abstand beizubehalten: Gehen Sie einen Schritt zurück, gewinnen Sie wieder Abstand und kommen Sie zur Ruhe.

Fallbeispiel:

Nachdem Nora innerlich Abstand gewonnen hatte und das starke Gefühl persönlicher Betroffenheit bei ihr langsam verschwunden war, begann sie in Ruhe, den Konflikt von vorne bis hinten zu analysieren: Wodurch war er entstanden? Wie hatte sie sich verhalten? Was hätte sie anders machen können?

\section{3 a) Worin besteht der Konflikt?}

Voraussetzungen für einen Konflikt sind:

- „Mindestens zwei Parteien

- Gemeinsame Berührungspunkte

- Unterschiedliche Ansichten hinsichtlich der

- Ziele

- Wege

- Verteilung von Ressourcen

- Gestaltung von Beziehungen

- Unterschiedliche Rollendefinitionen

- Unterschiedliche Beziehungserwartungen

- Gefühle“(Hintz 2011, S. 118). 
Die Ursachen für Konflikte liegen beispielsweise begründet in Verhaltensweisen wie einer Unterschätzung oder Geringschätzung. Jemand wird nicht für „voll“ genommen oder, mit anderen Worten z. B. von Ihnen aus einem Eltern-Ich-Zustand in einem KindIch-Zustand angesprochen. Negativer Stress, das Gefühl, von der Arbeitssituation überfordert zu sein, oder Missverständnisse können darüber hinaus dazu beitragen, Konflikte zu befeuern. Recht häufig sind nicht geklärte Machtfragen, mehr oder weniger offen ausgetragene Machtkämpfe, Konkurrenzdenken oder einfache Antipathien und damit verbunden eine nicht vorhandene Wertschätzung der anderen Person die Ursache. Auch nicht abschließend abgestimmte Ziele und Aufgaben, unterschiedliche Erwartungen, Schuldzuweisungen, Unterstellungen und Konkurrenzkämpfe können Konflikte befördern (vgl. Hintz 2011, S. 118). Neben unterschiedlichen Erwartungen entstehen Konflikte oft auch aus unausgesprochenen oder aus uneingestandenen Erwartungen.

Aufgrund ihres umwälzenden Charakters und der „Bedrohung“ bestehender Gewohnheiten und Privilegien ist es wenig verwunderlich, dass gerade im Umfeld von Innovation und Veränderung leicht Konflikte entstehen können.

Konflikte, die in Bibliotheken auftreten, können darüber hinaus auch andere Ursachen haben. Etwa können sie durch herausfordernde Situationen wie die folgenden entstehen:

- Überforderungsreaktionen auf zu viel Stress,

- Sparmaßnahmen,

- hoher Krankenstand,

- keine Nachbesetzung von vakanten Stellen,

- keine passende Eingruppierung,

- keine Honorierung herausragender Leistungen,

- unterschiedliche Werte,

- unterschiedliche Haltung zu Traditionen bzw. Innovationen,

- unterschiedliche Auffassungen über Prioritäten,

- unklares Führungsverhalten (mal Hü, mal Hott).

Es ist im nächsten Schritt gut, sich dezidiert darüber Gedanken zu machen, wer in einen Konflikt involviert ist.

- Ist es ein Konflikt zwischen oder innerhalb von Einzelnen, Gruppen oder der gesamten Organisation?

- Sind Personen unterschiedlicher Hierarchiestufen beteiligt?

- Sind nur die Personen an dem Konflikt beteiligt, zwischen denen er offensichtlich ausgebrochen ist?

- Sind im Hintergrund noch andere Personen am Konflikt beteiligt, die derzeit vielleicht nicht sichtbar sind, aber eventuell aus dem Verborgenen die Fäden ziehen?

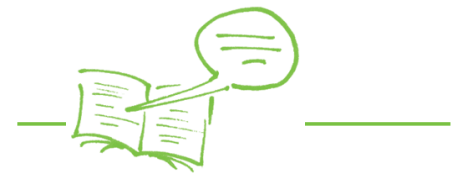

Zitat:

„Schlimmer noch und beharrlicher sind die Konflikte, die aus uneingestandenen Erwartungen entstehen. Sie haben vielleicht Erwartungen, derer Sie sich schämen, weil sie öffentliche Tabuzonen verletzen. Deshalb äußern Sie sie nicht, hoffen aber inständig, der andere möge sie doch ahnen. Und wenn er es nicht tut, strafen Sie ihn dafür bei günstiger Gelegenheit, die in den seltensten Fällen Bezug zum Gegenstand der Erwartungen hat. Etliche glauben auch, zu viel zu sagen und damit Selbstverständlichkeiten zu strapazieren. Aber diese Selbstverständlichkeiten gibt es nicht. Ich kenne keine Führungskraft, die zu viel und zu deutlich Erwartungen kommuniziert."

(Sprenger 2000, S. 237)

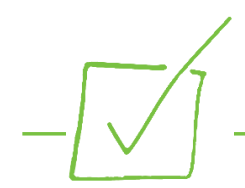

Checkliste: In den Konflikt involvierte Personen 

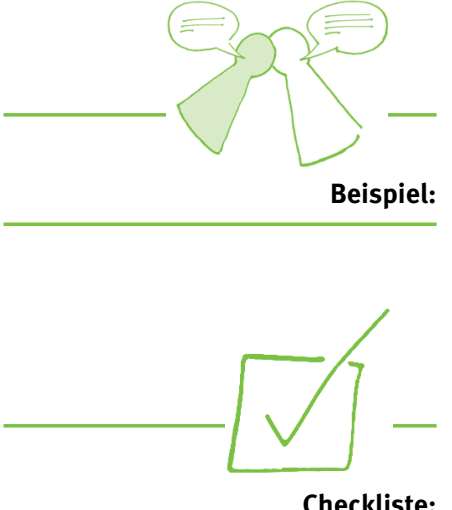

Mögliche Verhaltensweisen, die zur Entstehung oder Verschlimmerung eines Konflikts führen

\section{3 b) Wie ist der Konflikt entstanden?}

Es sind verschiedenste Verhaltensweisen, die dazu beitragen können, dass ein Konflikt entsteht oder dass er gravierender wird, als dies notwendig wäre. Hier können u.a. psychologische Aspekte eine Rolle spielen, die in diesem Buch bereits vorgestellt wurden.

\section{Fallbeispiel}

Der Konflikt mit Herrn 0. schwelte schon längere Zeit. Es wollte ihn nur keiner anpacken. Befeuert durch das in diesem Punkt passive Verhalten des Herrn Dr. Schache, haben auch alle anderen den Konflikt wie eine „heiße Kartoffel“ weitergereicht. Er ist nun durch das gemeinsame Gespräch das erste Mal direkt ans Tageslicht gekommen. Ein Aspekt, der dem Konflikt zugrundeliegt, ist sicherlich, dass Herr O. sich nicht mit seinen Fähigkeiten wertgeschätzt fühlt.

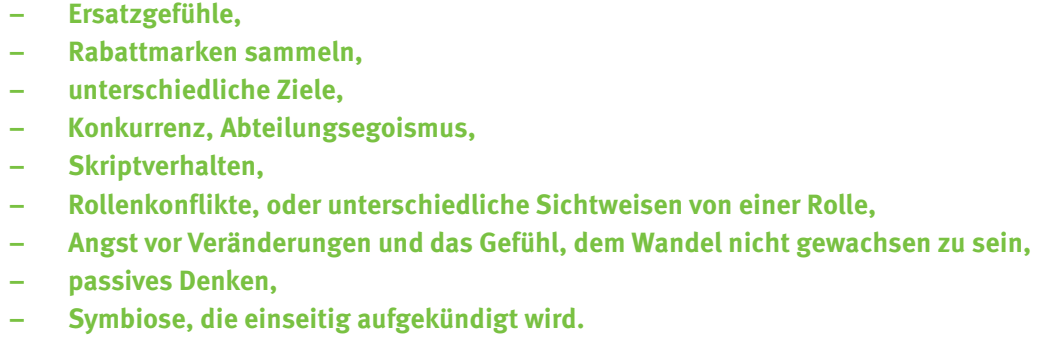

3 c) Wie habe ich mich verhalten? Wie haben andere Beteiligte sich verhalten? Das eigene Verhalten in einem bereits bestehenden Konflikt oder einem früher erlebten Konflikt zu analysieren, hilft, Verbesserungsmöglichkeiten für die Zukunft zu identifizieren.

\section{Fallbeispiel}

Nora reflektiert ihr Verhalten im Konflikt. Möglicherweise war es ungünstig, denkt sie jetzt, dass sie zu zweit das Gespräch gesucht haben, während Herr O. alleine war. Dadurch waren sie zahlenmäßig überlegen. Außerdem überlegt sie, dass sie Herrn 0. gegenüber vielleicht zu belehrend aufgetreten ist und dass sein trotziges Verhalten daher eventuell kein Wunder ist. 
In so einer Situation könnten Sie sich folgende Fragen stellen:

- Haben Sie sich passend zu Ihrer Rolle verhalten? Haben die anderen Beteiligten passend zu ihren Rollen agiert?

- Waren Sie „gut drauf"?

- Wie gestresst sind Sie derzeit? Bekommen Ihre verschiedenen Lebensbereiche genügend Beachtung? Schöpfen Sie daraus genügend Kraft?

- Waren Sie sich selbst Ihrer Ziele klar?

- Was haben Sie für Signale ausgesendet?

- Waren Sie in einem Skriptverhalten?

- Wie haben Sie kommuniziert? In welchem Ich-Zustand waren Sie? Haben Sie sich z. B. wie ein rebellisches Kind oder wie ein „Oberlehrer“ verhalten?

- Gibt es automatische Reaktionen, in die Sie leicht rutschen, wenn es im Gespräch schwierig wird und war dies hier der Fall?

- Hätten Sie sich anders verhalten können?

- Was hätten Sie besser machen können?

- Haben Sie gut zugehört?

- Haben Sie auf die nonverbalen Signale der anderen Beteiligten geachtet?

- Haben Sie gegenseitig Ihre Grenzen akzeptiert oder wurden Grenzen überschritten?
Jede Person hat ein eigenes Konfliktbewältigungsmuster. Kennen Sie das „Stück“, das eben in Ihrem Konflikt aufgeführt wurde oder handelt es sich um eine „Uraufführung"? Ist es Ihnen in ähnlicher Form schon öfter passiert, wie Sie sich in diesem Konflikt verhalten haben, handelt es sich möglicherweise um ein psychologisches Spiel bzw. Skriptverhalten.

4. Schritt: Was wünsche ich mir? Was würde mir jetzt gut tun?

Es ist hilfreich, sich nun zu fragen, was Sie sich selbst in dieser Situation wünschen.

Fallbeispiel:

Nora überlegt, was sie sich nun wünscht. Einerseits möchte sie ein gutes Verhältnis zu Herrn O. herstellen. Andererseits wünscht sie sich im Augenblick Ruhe und hat das Gefühl, noch nicht die Kraft für ein erneutes Gespräch und eine mögliche erneute Konfrontation zu haben.

Als nächstes ist es sinnvoll, sich selbst etwas Gutes zu tun, das man sich jetzt wünscht. So kann man gestärkt im Erwachsenen-Ich-Zustand in die Konfliktlösung starten: Was kann ich selbst tun, damit es mir gut geht?

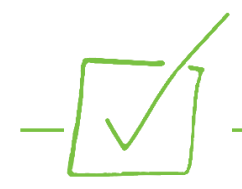

Checkliste:

Verhalten im Konflikt 


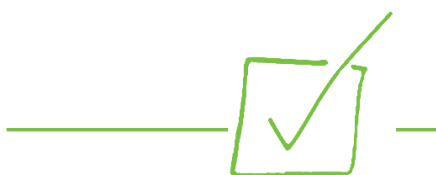

Checkliste: Was könnte mir jetzt gut tun?

- Heute früher Feierabend machen.

- $\quad$ Eine Runde spazieren gehen.

- Sport machen.

- $\quad$ Ein Eis essen gehen.

- $\quad$ Freunde treffen.

- Ins Kino gehen.

- Zur Massage gehen.

- Etwas Leckeres kochen.

- Eigene Ideen:

5. Schritt: Was kann ich tun, um den Konflikt beizulegen?

Bevor Sie wieder ins Gespräch einsteigen, ist es sinnvoll, dass Sie sich überlegen, welches Ziel Sie sich dafür setzen. Wie könnte ein erstes, realistisches Ziel lauten?

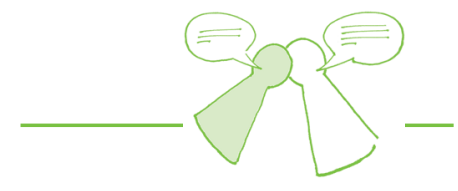

Fallbeispiel:

Nora entschließt sich, noch einmal das Gespräch mit Herrn 0. zu suchen. Dieses Mal alleine. Sie möchte zunächst die menschliche Ebene zwischen sich wieder in Ordnung bringen, bevor es an eine Lösung

Beispiel: des inhaltlichen Konflikts geht. Sie gesteht sich zu, dass dies etwas Zeit kosten wird und auch darf. 
Zunächst könnte es zur Lösung des Konflikts helfen, auf die andere Person aus dem Eltern-Ich-Zustand zuzugehen, unbedingte positive Zuwendung zu geben, eigene Wünsche zu äußern und Feedback zu geben. Auf der zwischenmenschlichen Ebene sollte klar werden: Du bist OK - Dein Verhalten nicht.

Anschließend könnte gemeinsam „Vertragsarbeit“ gemacht werden:

- Wie wollen wir die „Vertragsgestaltung“ zwischen uns machen, damit wir zukünftig eine gute Arbeitsbeziehung haben?

- Wie wollen wir mit dem Thema, das Inhalt unseres Konfliktes ist, umgehen?

- Was tun wir diesbezüglich?

- Wie könnte ein Weg hin zu einer Lösung aussehen?

\section{Schritt: Was kann ich zukünftig verändern?}

Ein aktueller oder ein durchstandener Konflikt kann mir wertvolle Lernmöglichkeiten für mein zukünftiges Verhalten aufzeigen.

Fallbeispiel:

Nora überlegt sich, dass zukünftig nur noch eine Person von ihnen ein 1:1-Gespräch mit Herrn 0. führen sollte, damit 0 . sich nicht so unter Druck gesetzt fühlt durch die „Übermacht“. Ferner nimmt sie sich vor, sich bei zukünftigen Situationen, bei denen sich bereits im Vorfeld ein gewisses Konfliktpotential abzeichnet, besser und bewusster darauf vorzubereiten.

Wenn Sie sich selbst auf eine eventuell schwierige oder konfliktreiche Situation vorbereiten wollen, was können Sie tun?

Gesprächsvorbereitung:

- Atmen! Tief ein- und ausatmen.

- Bewusstheit gewinnen, sich Klarheit verschaffen.

- Wo wollen Sie hin, ...? (Maximaler Effekt)

- Welche Kompromisse wären für Sie akzeptabel? (Minimaler Effekt)

- Fallen Ihnen noch weitere Lösungsmöglichkeiten ein, die den Interessen und Bedürfnissen beider Parteien entgegenkommen (Win-Win)

- Was wünschen Sie sich von Ihrem Gegenüber?

- Sich vorher bewusst machen, ob Sie in schwierigen Gesprächen zu bestimmten Verhaltensweisen neigen. Gibt es automatische Reaktionen, in die Sie allzu leicht verfallen, wenn es im Gespräch schwierig wird? Wenn Sie dies wissen, dann können Sie diese eher vermeiden.

- Klären, ob Ihr Gesprächspartner und Sie genug Zeit für das Gespräch haben.
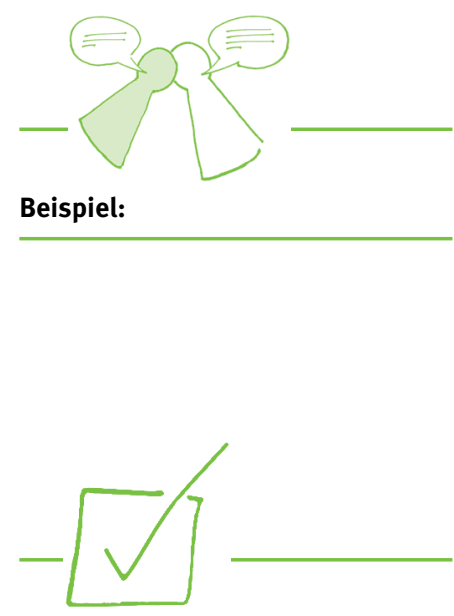

Checkliste ...

für schwierige bzw. wichtige Gespräche: 
- Hinfühlen, ob Sie jetzt wirklich bereit sind, das Gespräch zu führen.

- Sich vor dem Gespräch „sammeln“ und innerlich auf die bevorstehende Situation vorbereiten. Nicht dort hineinrauschen wie auf der Durchreise von einem Termin zum nächsten. Es ist jetzt besonders wichtig, dass Sie präsent sind, sich innerlich für mögliche Schwierigkeiten gewappnet haben und mit einer klaren Haltung in das Gespräch gehen. Nur dann können Sie sie auch klar artikulieren.

- Was erwarten Sie? Was erwartet vermutlich die andere Person?

Im Gespräch:

- Klären, wer welche Erwartungen hat.

- Wollen Sie zu Beginn des Gesprächs Regeln vereinbaren? Z. B.: Beide dürfen aussprechen, Gesprächsinhalte sind vertraulich, ...

- Welche guten Rahmenbedingungen können Sie schaffen, damit eine möglichst entspannte Atmosphäre entstehen kann (z. B. neutraler Raum, Störungsfreiheit, vielleicht ein Getränk)?

- Wie können Sie ggf. bei unterschiedlichen Hierarchiestellungen (Vorgesetzte / Mitarbeiterin bzw. Mitarbeiter) für das Konfliktgespräch einen ausbalancierten Machtausgleich schaffen, um ein positives und konstruktives Klima zu ermöglichen?

Was möchte ich zukünftig in (potentiellen) Konfliktsituationen verändern?

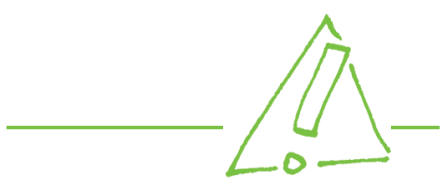

Tipps zum Mitnehmen aus dem Kapitel
- $\quad$ Eigene Klarheit hilft, Konflikten vorzubeugen und sie zu lösen.

- Klarheit fängt beim sich selbst definieren und abgrenzen an.

- Den eigenen Anteil bei Konflikten und Beziehungen selbstkritisch zu analysieren, ist Teil Ihrer Arbeit. - Vielfältige unterschiedliche psychologische Aspekte können dazu führen, dass Führungsbeziehungen sich schwierig gestalten und es zu Konflikten kommt.

\section{Weiterführende Literaturtipps}

Weiterführende Informationen u.a. zu Zuwendung, Widerstand, psychologischen Spielen sowie konkrete Tipps für die Gesprächsführung enthält: Gührs, Manfred; Nowak, Claus; Das konstruktive Gespräch - Ein Leitfaden für Beratung, Unterricht und Mitarbeiterführung mit Konzepten der Transaktionsanalyse; Limmer, Meezen, 6. Auflage, 2006

Informationen zu allen Inhalten der Transaktionsanalyse wie psychologischen Spielen, DramaDreieck, Rabattmarken usw.: Stewart, Ian; Joines, Vann; Die Transaktionsanalyse - Eine Einführung; Herder, Freiburg im Breisgau, 10. Auflage der Taschenbuchauflage, 2010

Eine ausführliche Darstellung psychologischer Spiele: Berne, Eric; Spiele der Erwachsenen - Psychologie der menschlichen Beziehungen; Rowohlt, Reinbek, 2. Auflage, 2002 